\title{
Adhesion Force Measurement of Mica surface using AFM
}

\author{
Chetan Subedi and SK Lamichhane \\ Department of Physics, Prithiv Narayan Campus, Tribhuvan University, Nepal \\ subedi_chetan@yahoo.com
}

\begin{abstract}
AFM is a tool to study the surface topography and associated molecular forces in real space image. AFM force-distance spectroscopy is the platform on which one can quantify atomic or molecular interactions and hence provide information that is critical to understand potential energy surface of mica through the measurement of pull-off force.
\end{abstract}

Keywords: AFM, force distance curve, nanoscale, pull off force.

\section{Introduction}

A group of minerals having perfect basal cleavage and capable of splitting into thin laminae is called mica. Chemically they contain complex silicate of aluminum and alkalis with hydroxyl. They crystallize in monoclinic system. Some varieties may contain iron, magnesium, lithium and rarely fluorine, barium, manganese and vanadium [1]. Mica is invaluable in the electrical industry because of its unique combination of physical, chemical and thermal properties, low power loss factor, dielectric constant and dielectric strength. Nowadays mica is finding increasing use in equipment that encounters very high temperatures like rockets, missiles and jet engine ignition system. It is reported that in telestar transmission satellites use of mica has been made [2].

Mica is invaluable in the electrical industry because of its unique combination of physical, chemical and thermal properties, low power loss factor, dielectric constant and dielectric strength. Dielectric strength is the ability to withstand high voltage without puncturing [3, 4].

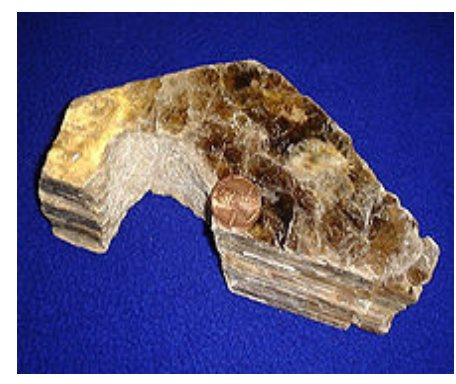

Mica sheet

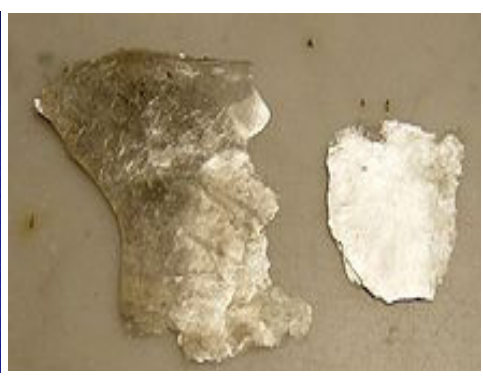

Mica flakes
Fig: 1 Atomic arrangement of muscovite mica
The AFM is one of the foremost tool for taking image and its manipulation matter at nanoscale. It was invented by Binning, Quate and Gerber in the year 1986 for which they were awarded Nobel prize. The study of materials and devices at nano scale has been a significant area of research in modern technology.The AFM however has the advantage of imaging almost any type of surface, including polymers, ceramics, composites, glass, and biological samples. The information is gathered by "feeling" the surface with a mechanical probe [5].

\section{Result and Discussion}

AFM is often used to obtain topography and chemical as well as mechanical properties of surfaces. In addition, AFM has capability to perform force measurements. The tip on the cantilever of the AFM is a force sensor. Consequently AFM can be utilized to perform the measurements of force distance curves, providing quantitative information on forces between the tip and the sample as a function of tip-sample distance [6].

Fig. 2 show a force/distance curve for silicon coated AFM-tip contacting a mica surface. The diagram shows the deflection of the AFM-cantilever as a function of distance between the mica surface and the AFM-tip, which is driven by a piezo. The deflection of the cantilever is detected in a split photo detector by the laser light reflected from the back of the cantilever. At the piezo-position labelled " 1 " the cantilever with the AFM-tip is force free and the corresponding force signal is set to zero. The tip remains free as long as the piezo approaches the mica surface. When reaching the 
close proximity of the mica surface a "jump-to-contact" occurs (at a distance of about $20 \mathrm{~nm}$ for the example given here, position "2").

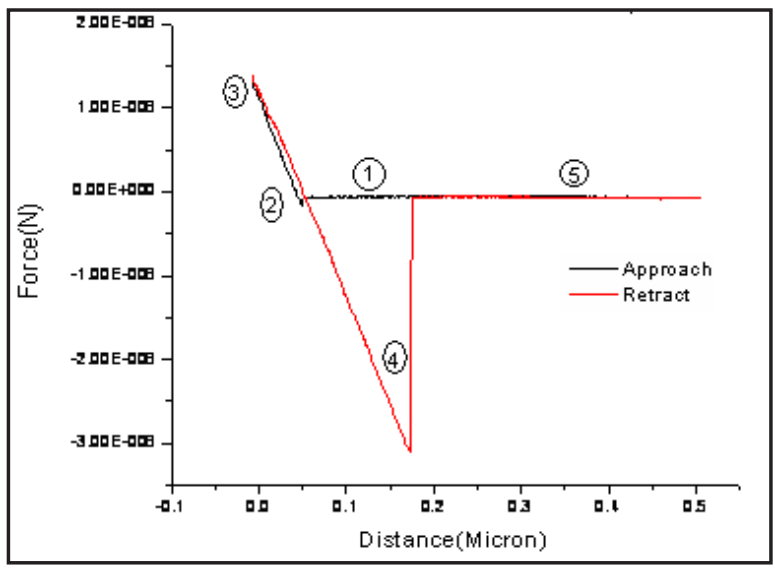

Fig. 2: Force-distance measurement. The graph shows the force measured by the AFM-cantilever versus tip sample distance.

At this distance the gradient of the attracting force between tip and mica surface becomes higher than the spring constant of the cantilever. The piezo drive pushes the AFM-tip further on/into the mica surface until point " 3 " is reached and the motion of the piezo is reversed. The pull off is continued until the tip comes free again (position " 5 "). The sudden pull off causes free vibrations of the cantilever. The force acting on the cantilever immediately before coming out of contact is a measure for the adhesion force between
AFM tip and surface [7]. In this study the adhesion force between the Si tip and the mica-surface measured at a piezo-position ("4") is $3.00 \mathrm{e}-008 \mathrm{nN}$ enforces to know about the potential energy surface.

\section{Conclusion}

Inter atomic force as a function of distance of separation between the moleculers. In AFM force-distance spectroscopy is the plate form on which one can quantify such atomic/molecular interaction and hence provide information that is critical to understand potential energy surfaces through the measurement of pull-off (adhesive) force. On the basis of measured pull-off force one can estimate the surface energy, work of adhesion, single atomic interaction etc.

\section{References}

[1] Mineralszone.com 2005

[2] Gem \& Mineral Miners, Inc, 2010, publisher?

[3] http//www.wikimedia foundation.org

[4] http//en wikipedia.org//wiki//file

[5] principle of AFM .pdf. Foxit reader pro 1.3/

[6] SK lamichhane, 2005. MEMS: response of a strained silicon semiconductor structure, $\mathrm{PhD}$ thesis JNU.

[7] Erich Santner and Bert Stegemann ,Federal Institute for Materials Research and Testing (BAM), Berlin, Germany. 